\title{
Distributed Detection Fusion Algorithms of Automatic Chemical Agent Detector
}

\author{
Alarm \\ Jin Gu ${ }^{1, a}$, Xuezheng Zhu ${ }^{2, b}$ \\ ${ }^{1}$ Postgraduate management team , Institute of NBC Defence, Beijing, China \\ ${ }^{2}$ Defense Equipment Department, Institute of NBC Defence, Beijing, China \\ aemail:gj8410@126.com, bemail:Zxz4x@aliyun.com
}

Keywords: toxic agent alarm, distributed, detection fusion, algorithms

\begin{abstract}
Whenever a chemical toxic agent attacks, there needs an accurate inspection to send alarm signals for the crew and protection equipment. Given the detection rate of single toxic agent warner is easier affected by the surroundings, the author has conducted a fusion algorithms on the principles of Bayes test and has confirmed that the false dismissal probability and false-alarm probability will decrease sharply after the fusion by numerical test.
\end{abstract}

Around a battlefield, the existence of a chemical attack forms the foundation of a chemical reconnaissance and protection. In other words, a precise judge of an uncertain chemical attack is rather important for the army to maneuver. Considering the current unsatisfying technique and more disturbing battlefield elements, single alarm has an undoubtedly weakness of false dismissal and false alarm. This distributed detection fusion technique allows every alarm send their detections around the location and the fusion center is able to make a general decision under a certain optimization principle according to the detections from every corner, which permits the detection range becoming wider and detection rate lower.

\section{General Description}

The distributed detection in battlefields is mainly a binary final system of determining 'yes' or 'no' in a battlefield. The numerical results of this detection are 0 or 1 . In this hypothesis detection, $\mathrm{H}_{1}$ means a yes-existence of the target and $\mathrm{H}_{0}$ means a no-existence.

The detection results of battlefield chemical detectors are obtained through different operators and equipment and stay separate from each other. Suppose the battlefield distributed toxic agent detection system is formed by one fusion center and $\mathrm{N}$ chemical detectors and the detection of every equipment is conducted individually. Given: $x_{i}$ as the value of toxic alarm, then the conditional probability under former conditions is $p\left(x_{i} \mid H_{i}\right), \mathrm{i}=0,1$. Suppose the empirical data are known for a certain value, then the points in the detection space will be formed under these data. We can set these two prior probabilities as $P_{0}$ and $P_{1}$. There are two typical mistakes in above two hypothesis detections as false dismissal and false-alarm ${ }^{[1]}$. Distributed detection fusion of toxic agent alarm is made to get more detection results and lower the rate of these two mistakes through fusion algorithms.

\section{Basic Numerical Calculation}

Individual toxic agent detector can only send the concentration around itself and set off the alarm when its concentration reaches the limitation. According to the alarm principle, the rate of false dismissal and false alarm is related to the current toxic concentration and humidity.

\subsection{False dismissal probability $-\mathbf{P}_{M}$}

(1) Affection from concentration

There are some collected data from previous studies about toxic agent false dismissal rate and 
toxic concentration value with the correspondent humidity around 58\%--62\%. The false dismissal and false alarm rate are counted as below.

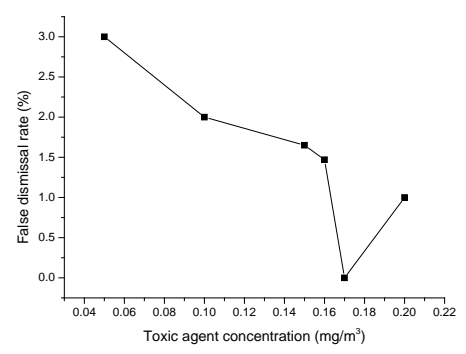

Figure 1 relation between false dismissal rate and toxic agent concentration

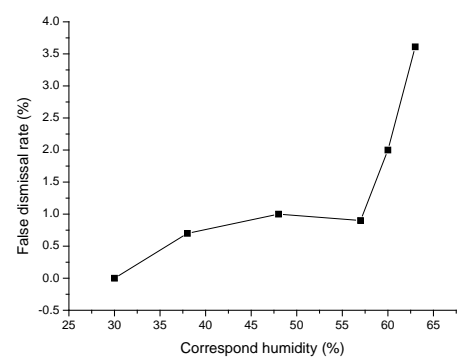

Figure 2 relation between false dismissal rate and correspond humidity

From Figure 1, we can find out that the false dismissal rate of toxic agent decreases with the high alarm rate under the same relative humidity. With the fitting data of the Gaussian curve, the expression of it is: $P_{M c}=a e^{-\frac{(x-b)^{2}}{c^{2}}}$.

By employing the Matlab curve fitting tool kit, the final results are: $a=3.276, b=0.004367$, $\mathrm{c}=0.1466$.

(2) Affection from humidity

Collect inspection data on an average toxic density of $0.2 \mathrm{mg} / \mathrm{m}^{3}$ first, then place them according to the relative humidity. This leads to Figure 2. The fitting data from $P_{M}^{*}=a e^{b x}$ are a=0.0001614, $b=0.1582$. From the curve, we can find that the false dismissal rate increases sharply when the humidity reaches a level. For example, when the humidity reaches $80 \%$, the false dismissal rate will increase to $50 \%$ in theory.

Considering density and humidity together, here is the expression between the false dismissal rate of toxic agent alarm and relative density and humidity:

C: toxic agent density around the alarm, $\mathrm{mg} / \mathrm{m}^{3}$

$$
P_{M}=P_{M c}+P_{M}^{*}=3.276 e^{-\left(\frac{c-0.00436}{(0.1666}\right)^{2}}+0.0001614 e^{0.1582 W}
$$

$\mathrm{W}$ : relative humidity in the location, \%

\subsection{False-alarm probability- $\mathbf{P}_{F}$}

When there are some tail gases like gasoline, diesel oil and kerosene in the air, they will form as interference for the final results ${ }^{[2]}$. As long as the density of these gases goes beyond, the toxic agents wouldn't be detected correctly. So we can regard it as a false alarm when the interference goes beyond $0.1 \%$. in order to simplify the calculating process, here is the false alarm rate expression of individual toxic agent alarm:

$$
P_{\mathrm{F}}=\left\{\begin{array}{l}
1 \frac{c(t)}{c(t)+C} \geq 0.1 \% \\
0 \frac{c(t)}{c(t)+C}<0.1 \%
\end{array}\right.
$$

$c(t)$ is the interference density in random occasions and represents the varied interference sources from battlefield which holds a deep relation with the interference source items. Suppose $c(t)$ meets the distribution regular of Gaussian interference, then it can be this one:

$$
c(t) \sim N\left(\mu, \sigma^{2}\right)
$$

Parameter $\mu$ and $\sigma$ are determined by the specific battlefield.

\subsection{Fusion algorithms design}

The local judge rules of battlefield point source toxic agent alarm are already completed within the equipment. Battlefield chemical hazard distributed detection fusion stays mainly in the optimization fusion principle. That means the most efficient way for improving false alarm and 
false dismissal is to optimize fusion with an already known local judge rule [3].

Considering there is a binary hypothesis inspection question with a supposed $H_{0}$ and $H_{1}$, and the prior probabilities for each are $P_{0}$ and $P_{1}$. There are $N$ alarm devices and the local judgment on the location toxic density is $u_{i}(i=1,2, \ldots, N)$. Among them:

$$
u_{i}=\left\{\begin{array}{r}
0 \text { if alarm i determines } H_{0} \text { tenable } \\
1 \text { if alarm i determines } H_{1} \text { tenable }
\end{array}\right.
$$

After the wholly formation of the local judge, it will be input to the fusion center, then the input will be $u_{i}(i=1,2, \ldots, N)$. Then we will get a general judge with the fusion rules in fusion center:

$$
u_{0}=\left\{\begin{array}{l}
0 \text { if determines } H_{0} \text { tenable } \\
1 \text { if determines } H_{1} \text { tenable }
\end{array}\right.
$$

The output results of each individual alarm can be regarded as a binary random variable. According to the previous discussion, $P_{\mathrm{F}}, P_{\mathrm{D}}, P_{\mathrm{M}}$ can represent the false alarm rate, inspection and false dismissal rate of detector $i^{[4]}$. They are $P_{F_{i}}=\operatorname{Pr}\left(u_{i}=1 \mid H_{0}\right), \quad P_{D_{i}}=\operatorname{Pr}\left(u_{i}=1 \mid H_{1}\right), \quad P_{M_{i}}=\operatorname{Pr}\left(u_{i}=0 \mid H_{1}\right)$.

If regard the judges of each alarm as the observation data, then this problem become a binary hypothesis inspection problem. The fusion rules are there to determine the minimum average cost in the fusion center. For every possible decisions there is a cost $C_{i j}, i=0,1, j=0,1 . C_{i j}$ means $H_{i}$ is the real cost when $H_{\mathrm{j}}$ exists. Then the Bayes risk function is as follows:

$$
\begin{aligned}
\mathfrak{R} & =\sum_{i=0}^{l} \sum_{j=0}^{l} C_{i j} P_{j} P\left(H_{i} \mid H_{\mathrm{j}} \text { 为真 }\right) \\
& =\sum_{i=0}^{l} \sum_{j=0}^{l} C_{i j} P_{j} \int_{R_{i}} p\left(y \mid H_{j}\right) d y
\end{aligned}
$$

Given: $R$ as the area of all observation data, then $R_{i}$ owns the same the decision area with hypothesis Hi. If the observation data is within area $R_{i}, H_{i}$ turns out to be correct. Expression (1) can be divided into two observing areas of $R_{0}$ and $R_{1}$.

$$
\begin{aligned}
& \Re=P_{0} C_{00} \int_{R_{0}} p\left(y \mid H_{0}\right) d y+P_{0} C_{10} \int_{R 1} p\left(y \mid H_{0}\right) d y \\
& +P_{1} C_{01} \int_{R_{0}} p\left(y \mid H_{1}\right) d y+P_{1} C_{11} \int_{R 1} p\left(y \mid H_{0}\right) d y
\end{aligned}
$$

As the points in observation area are produced on the known conditional probability, their cumulative distribution probability around the whole area is 1 . It can be also put into the expression $\int_{R} p\left(y \mid H_{j}\right) d y=1, j=0,1$. Expression (2) is as follows:

$$
\mathfrak{R}=P_{0} C_{10}+P_{1} C_{11}+\int_{R_{0}}\left\{\left[P_{1}\left(C_{01}-C_{11}\right) p\left(y \mid H_{1}\right)\right]-\left[P_{0}\left(C_{10}-C_{00}\right) p\left(y \mid H_{0}\right)\right]\right\} d y
$$

The former 2 conditions in expression (3) is fixed, which makes the latter condition have the power to control the minimum value of cost function $\mathfrak{R}$, It is clear that the cost of making wrong decisions is bigger than the ones for the right decisions [6]. So it is $C_{10}>C_{00}, C_{01}>C_{11}$. the minimum value of the previous expression can be shown as:

$$
\frac{p\left(y \mid H_{1}\right)}{p\left(y \mid H_{0}\right)} \underset{H_{0}}{<} \frac{P_{0}\left(C_{10}-C_{00}\right)}{P_{1}\left(C_{01}-C_{11}\right)} \triangleq \eta
$$

$\eta$ represents the threshold. Basing on the definition of holistic decision, the left part of the previous expression can be wrote as: 


$$
\begin{aligned}
\frac{p\left(y \mid H_{1}\right)}{p\left(y \mid H_{0}\right)}=\frac{P_{r}\left(u_{1}, u_{2}, \cdots, u_{N} \mid H_{1}\right)}{P_{r}\left(u_{1}, u_{2}, \cdots, u_{N} \mid H_{0}\right)} & =\prod_{i=1}^{N} \frac{P_{r}\left(u_{i} \mid H_{1}\right)}{P_{r}\left(u_{i} \mid H_{0}\right)} \\
& =\prod_{R_{1}} \frac{P_{r}\left(u_{i}=1 \mid H_{1}\right)}{P_{r}\left(u_{i}=1 \mid H_{0}\right)} \prod_{R_{0}} \frac{P_{r}\left(u_{i}=1 \mid H_{1}\right)}{P_{r}\left(u_{i}=1 \mid H_{0}\right)} \\
& =\prod_{R_{1}} \frac{1-P_{M_{i}}}{P_{F_{i}}} \prod_{R_{0}} \frac{P_{M_{i}}}{1-P_{F_{i}}}
\end{aligned}
$$

Substitute expression (5) for (4), then conduct a logarithmic operation.

$$
\begin{gathered}
\sum_{R_{1}} \ln \frac{1-P_{M_{i}}}{P_{F_{i}}}+\sum_{R_{0}} \ln \frac{P_{M_{i}}}{1-P_{F_{i}}} \stackrel{\substack{u_{0}=1 \\
u_{0}=0}}{>} \ln \eta \\
\sum_{i=1}^{N}\left[u_{i} \ln \frac{\left(1-P_{M_{i}}\right)}{P_{F_{i}}}+\left(1-u_{i}\right) \ln \frac{P_{M_{i}}}{1-P_{F_{i}}}\right]_{u_{0}=0}^{>}>\ln \eta
\end{gathered}
$$

From that, the final fusion decision rules can be realized by comparing the values of both sides.

$$
\sum_{i=1}^{N} u_{i}\left[\ln \frac{\left(1-P_{M_{i}}\right)\left(1-P_{F_{i}}\right)}{P_{M_{i}} P_{F_{i}}}\right] \underset{u_{0}=0}{\stackrel{u_{0=1}}{>}} \ln \left[\eta \prod_{i=1}^{N}\left(\frac{1-P_{F_{i}}}{P_{M_{i}}}\right)\right]
$$

When the left side of the expression is higher than the right, the fusion output is $u_{0}=1$, which means $H_{1}$ is tenable and the chemical attack exists. Or the $H_{0}$ is tenable showing an zero existence of chemical attack.

\section{Numerical Simulation Experiment}

Summing up the previous calculations, this numerical simulation experiment has four steps. First is to produce the relative density response of the appointed area. Then generate the local judge decisions of each local alarm according to the history data. These data need to be sent to the fusion center. Finally get to the final fusion decision through the judge rules of the fusion center.

\subsection{Background setting}

As for the judgment for a battlefield chemical attack, false dismissals are more dangerous than false alarm, which reminds that the false dismissal should be considered more in the simulation. This directly relates to the correct response rate of the toxic agent alarm when there is a chemical attack. The only necessary thing is the simulation for generating certain toxic density. The diffusion mode adopts Gaussian puff model of fixed wind. The ten alarms are $A_{1}, A_{2}, \ldots A_{10}$, and their coordinates are on the $l=500 \mathrm{~m}$ line away from the chemical weapons' explosive points. They are 1 meter away from each other. The ordinate of $A_{i}$ is $y_{i}=5.5-i$.

In a short duration after the chemical attack, a toxic cloud cluster is released. Basing on Lachterman turbulent diffusion equation, we can get a density forecast on the leeward. Here is the expression of any point.

$$
C(x, y, z, t)=\frac{Q_{0}}{4 \pi K_{0} t\left(K_{1} n^{2} z_{1}^{(n-2)} t\right)^{1 / n} \Gamma(1+1 / n)} e^{-\left[\frac{(x-u t)^{2}+y^{2}}{4 K_{0} t}+\frac{z^{n}}{K_{1} n^{n} z_{1}^{n-2} t}\right]}
$$

Given the initial conditions: $\mathrm{Q}_{0}=50 \mathrm{~kg}$, non-isothermal condition, $K_{0}=1.785, K_{1}=0.014, n=1.10$, $\Gamma(1+1 / n)=0.96523$, height of $\mathrm{Z}=1 \mathrm{~m}$ and toxic cloud cluster moves on the $\mathrm{X}$ axis.

\subsection{Algorithms realization}

Suppose the current battlefield relative humidity is $70 \%$. According to the densities in table 1 and fitting expression of false dismissal rate, every alarm can get an accurate result of them, which false alarm rate relates to the random interference signals in the battlefield. For threshold value $\eta=\frac{P_{0}\left(C_{10}-C_{00}\right)}{P_{1}\left(C_{01}-C_{11}\right)} \quad\left(C_{11}=C_{00}=0, C_{10}=C_{01}=1\right)$, the cost for the right decision is 0 while the cost for the wrong is 1 . For the prior probability, given $P_{0}=P_{1}=50 \%$, we can get a local judge result by using the 
Matlab random function to produce 10,000 groups from $A_{1}-A_{10}$, then combine with expression (6) to get the final fusion decision result to get the false dismissal rate. Random matrix can be realized through randsrc (m, $\mathrm{n}$, [alphabet; prob])function. Among it, $m$ and $n$ represents the generated matrix dimension, 'alphabet' represents the random numbers and 'prob' represents the occurrence probability of each random number. The simulation results for false dismissal and false alarm rate are shown in table 1 and 2.

Table 1 simulation results for false dismissal rate

\begin{tabular}{|l|l|l|l|l|l|l|l|l|l|l|l|}
\hline $\begin{array}{l}\text { experiment } \\
\text { times }\end{array}$ & 1 & 2 & 3 & 4 & 5 & 6 & 7 & 8 & 9 & 10 & $\begin{array}{l}\text { average } \\
\text { value }\end{array}$ \\
\hline $\begin{array}{l}\text { fusion false } \\
\text { dismissal } \\
\text { rate \% }\end{array}$ & 0.01 & 0.04 & 0.01 & 0.02 & 0.01 & 0.03 & 0.02 & 0.02 & 0.03 & 0.01 & 0.02 \\
$\begin{array}{l}\text { original false } \\
\text { dismissal } \\
\text { rate \%(min) }\end{array}$ & 12.61 & 10.66 & 10.48 & 10.84 & 10.22 & 9.85 & 10.60 & 9.98 & 10.65 & 9.97 & 10.586 \\
\hline
\end{tabular}

Same as before, given a zero chemical attack first, then generate random numbers through fixed false alarm rate $P_{\mathrm{F}}=5 \%$. With the final fusion calculation, here is the specific result.

Table 2 simulation results for false alarm rate

\begin{tabular}{|l|l|l|l|l|l|l|l|l|l|l|l|}
\hline $\begin{array}{l}\text { experiment } \\
\text { times }\end{array}$ & 1 & 2 & 3 & 4 & 5 & 6 & 7 & 8 & 9 & 10 & $\begin{array}{l}\text { average } \\
\text { value }\end{array}$ \\
\hline $\begin{array}{l}\text { fusion false } \\
\text { dismissal } \\
\text { rate \% }\end{array}$ & 0.01 & 0.02 & 0.01 & 0.01 & 0.01 & 0.01 & 0.02 & 0.01 & 0.01 & 0.02 & 0.014 \\
\hline $\begin{array}{l}\text { original false } \\
\text { dismissal } \\
\text { rate \%(min) }\end{array}$ & 5.03 & 4.63 & 5.04 & 4.97 & 5.06 & 5.14 & 5.06 & 4.63 & 4.98 & 4.99 & 4.953 \\
\hline
\end{tabular}

\subsection{Discussion}

In one word, with the same battlefield condition, the false dismissal rate sharply decreases with a fusion calculation from an average $10.586 \%$ to $0.02 \%$. Benefit from this, false alarm rate decreases greatly from an average $4.953 \%$ to $0.014 \%$. Through this distributed detection fusion calculation in this paper, the alarm accuracy can be greatly improved by several alarms working and fusing together under a stable equipment performance. This provides a fundamental work for instructors making right decisions.

\section{References}

[1] Zhengxin Liu, Nan Yao. Probability and mathematical statistics [M]. Hubei Science \& Technology Press, 2005.10.

[2] Jun Ji, Qibin Huang, Xuequan Ding. Foreign Chemical Agent Detector Technology and Reviews [M]. Beijing: National Defense Industry Press, 2016.04.

[3] Xuehai Hu, Houjun Wang, Jianguo Huang. Final Optimization Algorithms of Distributed Target Detection Fusion [J]. Journal of University of Electronic Science and Technology of China, 2013, 42(3): 375-379.

[4] Viswanathan R, Varshney P K. Distributed detection with multiple sensors I. Fundamentals[J]. proceedings of the IEEE.1997.85(1):54-63.

[5] Guo H, Low K, Nguyen H. Optimizing the Localization the Localization of a Wireless Sensor Network in Real Time Based on a Low-Cost Microcontroller[J]. Ndral Lron Ranaon on, 2011,58(3):741-749

[6] Guerriero M, Svensson L, Willett P. Bayesian Data Fusion for Distributed Target Detection in Sensor Networks[J]. IEEE Transactions on Signal Processing, 2010,58(6):3417-3421 